\title{
Diversificación de exportaciones y crecimiento económico: evidencia empírica para Chile y Colombia (1980-2015)
}

\author{
AlmanZA-RAmíreZ, CAMilo \\ Universidad del Norte (Colombia) \\ Correo electrónico: almanza@uninorte.edu.co \\ RODRÍGUEZ-AlBOR, GUSTAVO JESÚS \\ Universidad Autónoma del Caribe (Colombia) \\ Correo electrónico: grodrigu30@cuc.edu.co \\ Gómez-Pacheco, Álvaro \\ Universidad del Norte (Colombia) \\ Correo electrónico: gomezaa@uninorte.edu.co \\ VERBEL-MONTES, IVÁN \\ Universidad del Norte (Colombia) \\ Correo electrónico: iverbel@uninorte.edu.co
}

\begin{abstract}
RESUMEN
La relación entre las exportaciones y el crecimiento económico ha sido ampliamente discutida en la literatura, aunque actualmente no existe un consenso claro sobre el tema. En este artículo se evalúa la hipótesis de que la diversificación de exportaciones está vinculada al crecimiento económico a través de externalidades del tipo aprender haciendo y aprender exportando, mediante el uso de métodos multivariantes para series de tiempo -modelos VAR y VEC- con datos de exportaciones de Chile y Colombia para el periodo 1995-2015. La prueba Dickey-Fuller aumentada se usa para evaluar la estacionariedad de las series. El test de Johansen se emplea para determinar el grado de cointegración entre las variables, y la prueba de causalidad Granger para establecer la relación de causalidad entre las mismas. Los resultados muestran que el crecimiento basado en la diversificación de exportaciones es relevante para Chile, mientras que para Colombia se detectó una relación de causalidad que va del crecimiento económico a la diversificación de exportaciones.
\end{abstract}

Palabras clave: diversificación de exportaciones, crecimiento económico, ventaja comparativa revelada.

Clasificación JEL: C10; F10; O10.

MSC2010: 91B60; 91B62; 91B84.

Artículo recibido el 7 de mayo de 2018 y aceptado el 10 de mayo de 2019. 


\title{
Export diversification and economic growth. Empirical: evidence for Chile and Colombia (1980-2015)
}

\begin{abstract}
The relationship between exports and economic growth has been widely discussed in the literature, although there is currently no clear consensus on the subject. This article evaluates the hypothesis that the exportdiversification is linked to economic growth through externalities of the type learning by doing and learning by exporting, by using multivariate methods for time series -VAR and VEC models- with exports's data from Chile and Colombia for the period 1995-2015. The Dickey-Fuller Augmented test is applied to check the series's stationarity. The Johansen test is used to determine the degree of cointegration between the variables, and the Granger causality test to evaluate the causality relationship between them. The results show that the export diversification-led growth is relevant for Chile, while for Colombia a causal relationship that goes from economic growth to export diversification was detected.
\end{abstract}

Keywords: exports' diversification, economic growth, revealed comparative advantage.

JEL classification: C10; F10; O10.

MSC2010: 91B60; 91B62; 91B84.

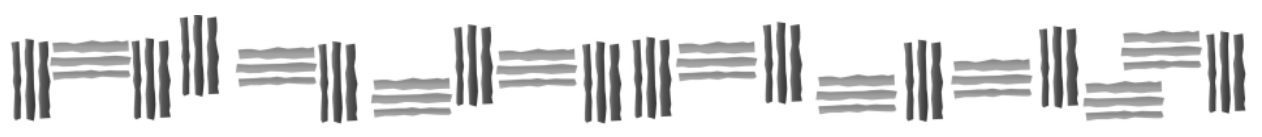




\section{Introducción.}

El desarrollo económico es un proceso complejo de profundas transformaciones que suele requerir, en términos de la estructura económica de un país, el tránsito desde la producción y exportación de bienes primarios y materias primas hacia bienes manufacturados y con alto contenido tecnológico. De esa complejidad deriva una pluralidad de criterios en la literatura especializada que buscan explicar la relación entre el comportamiento de las exportaciones y el crecimiento económico.

Así, la investigación en torno a esa relación versa sobre cuatro posibles resultados (Chen, 2007). Uno de ellos plantea la existencia de una relación de causalidad unidireccional que va de las exportaciones al crecimiento económico (export-led growth hypothesis). Aquí, el impacto en el crecimiento del producto se origina a través de incentivos a los productores nacionales para usar mejores técnicas de producción y a ser más competitivos en el mercado mundial (Gokmenoglu, Sehnaz, \& Taspinar, 2015; Balaguer \& Cantavella-Jordá, 2004; Abdulai \& Jaquet, 2002; Awokuse, 2003; Hye, Wizarat \& Lau, 2013). Por el contrario, otros autores proponen la existencia de una relación causal unidireccional que va del crecimiento económico al incremento de las exportaciones (Panas \& Vamvoukas, 2002; Jin, 2002). Algunas explicaciones arguyen una relación bidireccional entre las exportaciones y el crecimiento del producto, mientras otra corriente postula la existencia de una relación neutral entre esas dos variables (Chen, 2007).

En ese contexto se observa que en muchos países en vías de desarrollo las exportaciones contribuyen significativamente al aumento del ingreso per cápita a largo plazo. Muchos países que dependen de las exportaciones de materia prima o tienen una limitada cesta exportadora sufren por la inestabilidad de sus exportaciones y por el deterioro en los términos de intercambio -tesis Prebisch-Singer-. Los movimientos de la tasa de cambio pueden torpedear los esfuerzos hechos en materia de planificación económica, reducir la demanda por importaciones y contribuir a la generación de déficit de inversión debido a los altos niveles de riesgo (Herzer \& Nowak-Lehnmann, 2006; Dawe, 1996; Bleaney \& Greenaway, 2001). Como alternativa, la ampliación de la cesta exportadora puede contribuir a la reducción del riesgo (Acemoglu y Zilibotti, 1997) y a mejorar los términos de intercambio (Stanley \& Bunnag, 2001). La diversificación de las exportaciones favorece la "graduación" de los países, al pasar del estatus en vía de desarrollo (Least Developed Countries, LDC) a desarrollados (Developed Countries, DC), apoyados en la evidencia aportada por Michaely (1977) y Moschos (1989) en la que se muestra la relación entre las exportaciones y los growth kicks (Gutiérrez de Piñeres \& Ferrantino, 1997). Existe, por lo tanto, una importante correlación entre la diversificación y la estabilidad de los ingresos por exportaciones y la sostenibilidad del crecimiento económico (Stanley \& Bunnag, 2001).

Sin embargo, la aceleración del comercio transfronterizo y la globalización exponen las exportaciones a las dinámicas de los mercados internacionales, exigiendo competitividad como aspecto clave del éxito en la diversificación. Esas dinámicas competitivas tienen un efecto acelerador sobre la generación de conocimiento al interior de los sectores exportadores que, en su mayoría, resultan de externalidades del tipo learning by exporting y learning by doing. El objetivo de este artículo es, precisamente, examinar la hipótesis de que la diversificación de las exportaciones está vinculada con el crecimiento económico a través de esas externalidades, siguiendo la modelación sugerida por Herzer y Nowak-Lehnmann (2006). En este esfuerzo por contrastar dichos vínculos, las unidades de análisis corresponden a economías enmarcadas en intensos procesos de apertura $\mathrm{y}$, para el caso, con importantes diferencias en su dinámica comercial, siendo representativas de ello las economías de Chile y Colombia. El período de estudio incluye información relevante de más de tres décadas y se extiende entre 1980 y 2015.

El artículo incluye, posterior a este apartado introductorio, una revisión de la literatura seguida por la presentación del modelo empírico a emplear. Una penúltima sección describe los 
datos y la metodología econométrica implementada y, finalmente, se exponen los resultados y las conclusiones del estudio.

\section{Revisión de literatura.}

La diversificación de las exportaciones no necesariamente es producto de las ventajas comparativas de los países en sentido ricardiano. Los costos de descubrimiento a los que se enfrentan los emprendedores están asociados a la incertidumbre en la producción de nuevos bienes; si los emprendedores tienen éxito, las ganancias son socializadas (un spillover de información) mientras que en caso contrario la pérdida es totalmente privada, lo que deriva en un déficit de inversión en nuevas actividades económicas. De este modo, para Hausmann y Rodrik (2003) el rol de los gobiernos en la promoción del crecimiento de la industria y el cambio estructural reside en la promoción del emprendimiento y la creación de un marco de incentivos para que los emprendedores inviertan en nuevas actividades económicas. Tomando como base los costos de descubrimiento, Hausmann, Hwang y Rodrik (2006) encuentran que los países que producen bienes con alta productividad disfrutan de un crecimiento económico más rápido que aquellos con bienes de baja productividad.

La ampliación de la base exportadora es producto de la innovación dirigida hacia la introducción de nuevos sectores en la economía, precedida de un esfuerzo creativo; el descubrimiento de nuevos bienes implica entonces la generación de spillovers de conocimiento, que derivan en externalidades relacionadas con un proceso de acumulación de conocimiento proclive al crecimiento económico. En tal sentido, Romer (1990) considera que la diversificación puede ser vista como un factor de producción que promueve la eficiencia de otros insumos.

En línea con lo anterior, Al-Marhubi (2000) argumenta que las externalidades asociadas con la diversificación de las exportaciones siguen un proceso similar al learning by doing propuesto por Arrow (1962), en el que la acumulación de conocimiento acerca de mejores formas de producir es un producto no planeado de la acumulación de capital y el proceso de producción. Por su parte, Hausmann y Klinger (2006) sostienen que la transformación estructural de las economías depende de los bienes exportables que se encuentren cerca de otros bienes con una mayor sofisticación y alto valor. De este modo, lo deseable para cada país es encontrar una densidad del espacio de productos muy cercana a sus posibilidades de producción, en tanto que se configura un spillover de conocimiento en la medida que la diversificación de las exportaciones ocurre cuando existen procesos de learning by doing.

En la investigación empírica se han empleado diversas técnicas para explorar la relación existente entre diversificación de exportaciones y crecimiento económico. Al-Marhubi (2000), usando un modelo de mínimos cuadrados ordinarios estimado con datos de una muestra transversal para 91 países, encuentra evidencia a favor del crecimiento económico liderado por la diversificación de las exportaciones. De forma similar, en las islas Mauricio, Sannassee, Seetanah y Lamport (2014) mediante la estimación de un vector de cointegración encuentran una relación positiva, aunque débil tanto en el corto como el largo plazo, entre la diversificación de las exportaciones y el crecimiento económico.

Por su parte, Misztal (2011) utilizando un Modelo Vectorial Autorregresivo (VAR), encontró que para los países integrantes de la Unión Europea durante el periodo 1995-2009, la diversificación fue uno de los factores más importantes en la determinación del nivel del Producto Interno Bruto (PIB) per cápita. A su vez, Balaguer y Cantavella-Jordá (2004), utilizando técnicas de cointegración y análisis de causalidad con datos de España para el periodo 1961-2000 obtuvieron evidencia de la existencia de una relación de largo plazo entre la producción, la expansión de las exportaciones y el cambio estructural de las mismas. Sus resultados indican que las exportaciones son un factor determinante para la producción real. 
Además, encuentran que el crecimiento económico refuerza la expansión de las exportaciones, y concluyen que existe una causalidad inversa entre estas dos variables.

En Latinoamérica diversos estudios abordan el tema. Se encuentra, por ejemplo, que en las relaciones de intercambio comercial entre la economía uruguaya y países como Argentina y Brasil, analizadas por Mordecki y Piaggio (2008), la demanda externa actúa como principal determinante de las exportaciones y su diversificación. Se encuentra también, en lo referente a la política de diversificación de exportaciones -mediante un estudio realizado por Ruiz y Vera (2013) en Perú- que la política de diversificación debe ir acompañada de un encadenamiento productivo comercial que contribuya a la interacción y creación productivistas a través de los incentivos.

La experiencia centroamericana muestra que la diversificación cumple con un papel estabilizador de ingresos. En Costa Rica, El Salvador, Honduras y Guatemala, Stanley y Bunnag (2001) evaluaron si la diversificación de exportaciones había disminuido la volatilidad de los ingresos externos, llegando a la conclusión de que a pesar de los esfuerzos aún predominaban los sectores tradicionales y que pocos productos manufacturados habían estabilizado sus ingresos más que en la diversificación de productos agrícolas.

Asimismo, en Chile, los trabajos en torno a la relación entre diversificación de exportaciones y crecimiento económico ratifican lo expuesto por algunas de las principales corrientes de estudios sobre el tema. El análisis histórico de las exportaciones realizado por García, Meller y Repetto (1996) concluye que se debe estimular la expansión de los sectores domésticos no exportadores para que sean integrados al comercio internacional. Gutiérrez de Piñeres y Ferrantino (1997) sostienen que la diversificación ha sido una consecuencia de las políticas de liberalización de la economía, mientras que Agosin (1999), encontró que la política de liberalización fue el principal eje de dinamismo de integración e intercambio de la economía chilena. Herzer y Nowak-Lehnmann (2006) por su parte, mediante la estimación de una función Cobb-Douglas aumentada y utilizando series de tiempo, encontraron que la diversificación de las exportaciones ha jugado un papel importante en el crecimiento económico chileno.

Finalmente, en Colombia, Gutiérrez de Piñeres y Ferrantino (1999) encontraron evidencia de la existencia de una relación causal que va del crecimiento económico hacia el crecimiento de las exportaciones. Es decir, el crecimiento de las exportaciones es explicado por la aceleración del PIB, y Mejía (2011) sostiene que el éxito de la implementación de la diversificación de exportaciones depende del énfasis en la participación de la pequeña y mediana empresa para incrementar la probabilidad de exportar mayores productos -no necesariamente de manufactura- hacia el exterior.

\section{Evolución de las importaciones en Chile y Colombia.}

De acuerdo con la información reportada por el Banco Mundial, Chile ha mostrado un importante crecimiento en el grado de apertura con respecto al conjunto de su actividad económica a partir de 1974, alcanzando su coeficiente de apertura un valor máximo $(0,81)$ en el 2007. Este comportamiento refleja claramente una política económica orientada al comercio internacional. Colombia ha mostrado un grado de apertura moderado con respecto a Chile, con un índice de apertura, pues a partir de 1990 oscila entre 0.33 y 0.39 (Gráfico 1).

En cuanto a exportaciones de bienes, la Comisión Económica para América Latina y el Caribe (CEPAL) registra, en el caso colombiano, una disminución de las exportaciones de manufacturas con respecto a las exportaciones totales. La reducción fue de $17.5 \%$ en el año 2014 con respecto al promedio de $34.8 \%$ registrado entre los años 2000-2010 (Gráfico 2). 
Gráfico 1. Coeficiente de apertura económica, 1980-2015 (como porcentaje del comercio total respecto al PIB).

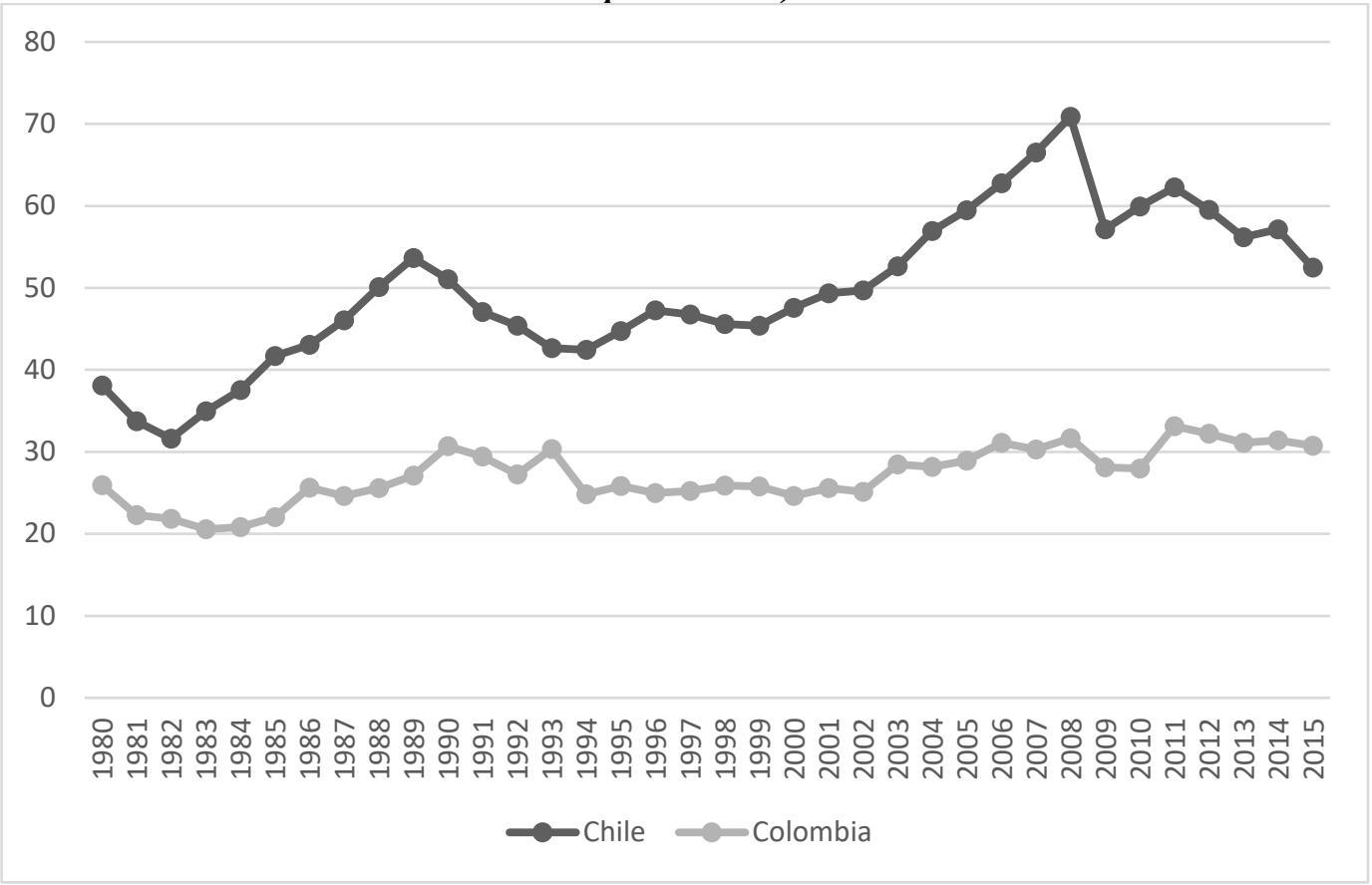

Fuente: Banco Mundial (2017). Banco de datos Indicadores de desarrollo mundial.

Gráfico 2. Composición de las exportaciones de bienes en Chile, 1980-2015 (en porcentajes respecto al total).

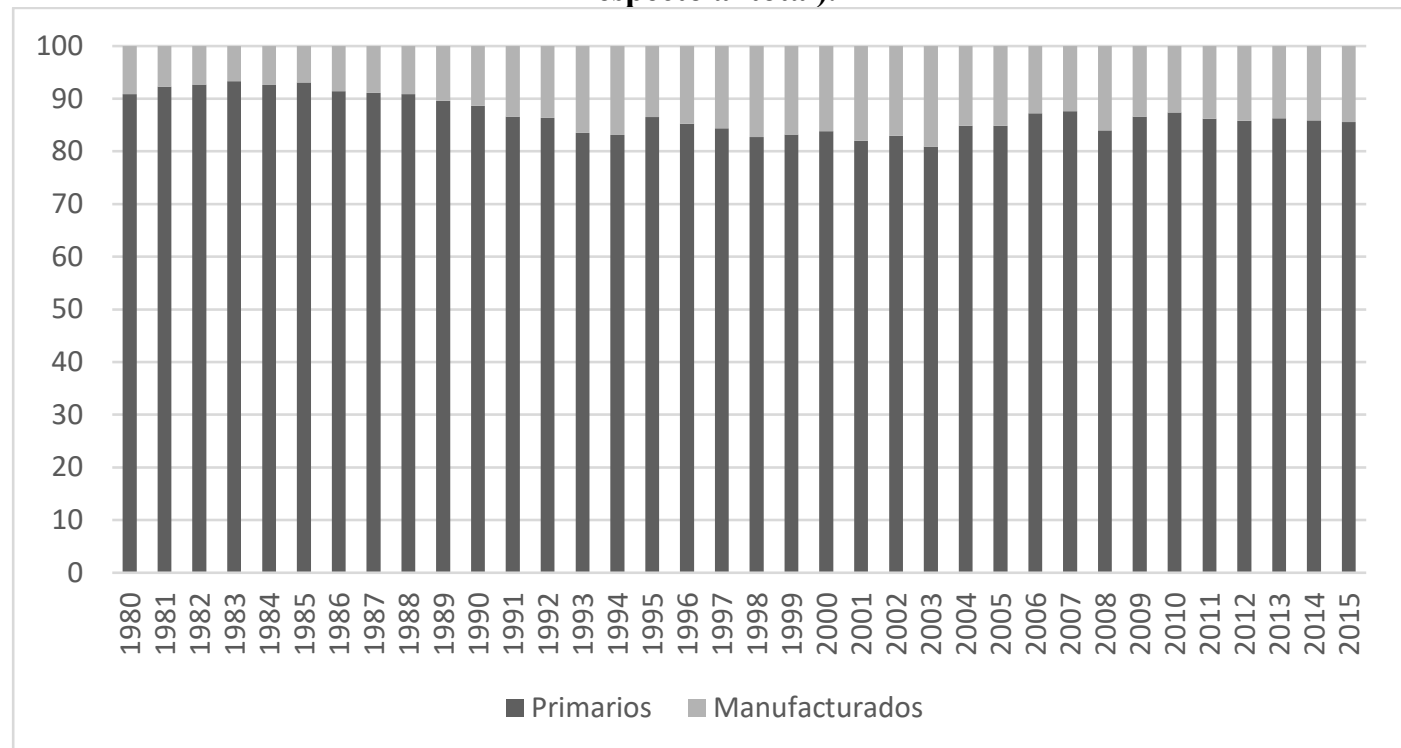

Fuente: Cepalstat (2017). Base de datos de Estadística e indicadores.

En el caso de Chile, aunque también se ha evidenciado una disminución en la proporción de exportaciones de manufacturas frente al total de exportaciones, este indicador se ha mantenido cerca de su valor histórico de 14.9\% para el período entre 1990-2014 (Gráfico 3).

En lo que respecta a diversificación de exportaciones para Chile y Colombia, ambos países presentan una tendencia decreciente en el Índice de Diversificación (especialización) de Exportaciones, lo que evidencia que ambas economías están desarrollando procesos de diversificación de sus exportaciones (Gráfico 4). 
Gráfico 3. Composición de las exportaciones de bienes en Colombia 1980-2015 (en porcentajes respecto al total).

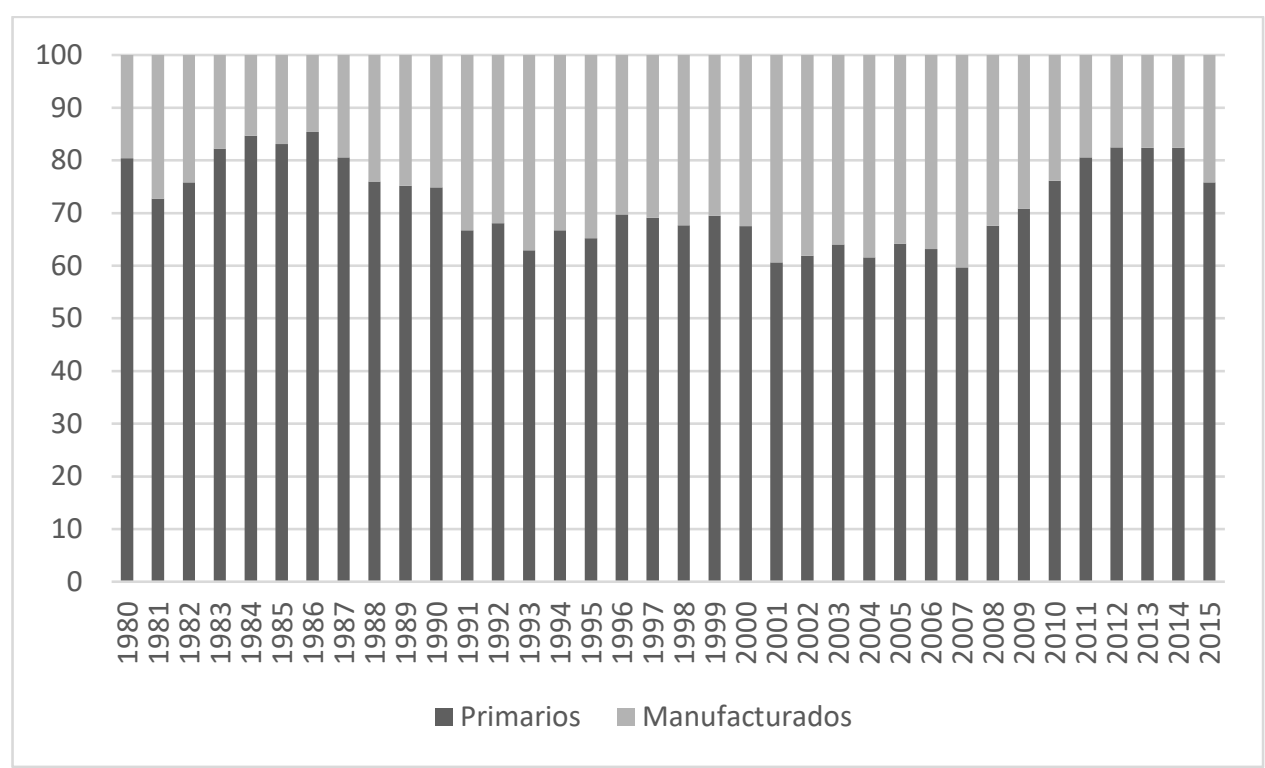

Fuente: Cepalstat (2017). Base de datos de Estadística e indicadores.

Gráfico 4. Índice de Diversificación (especialización) de Exportaciones (1980-2015).

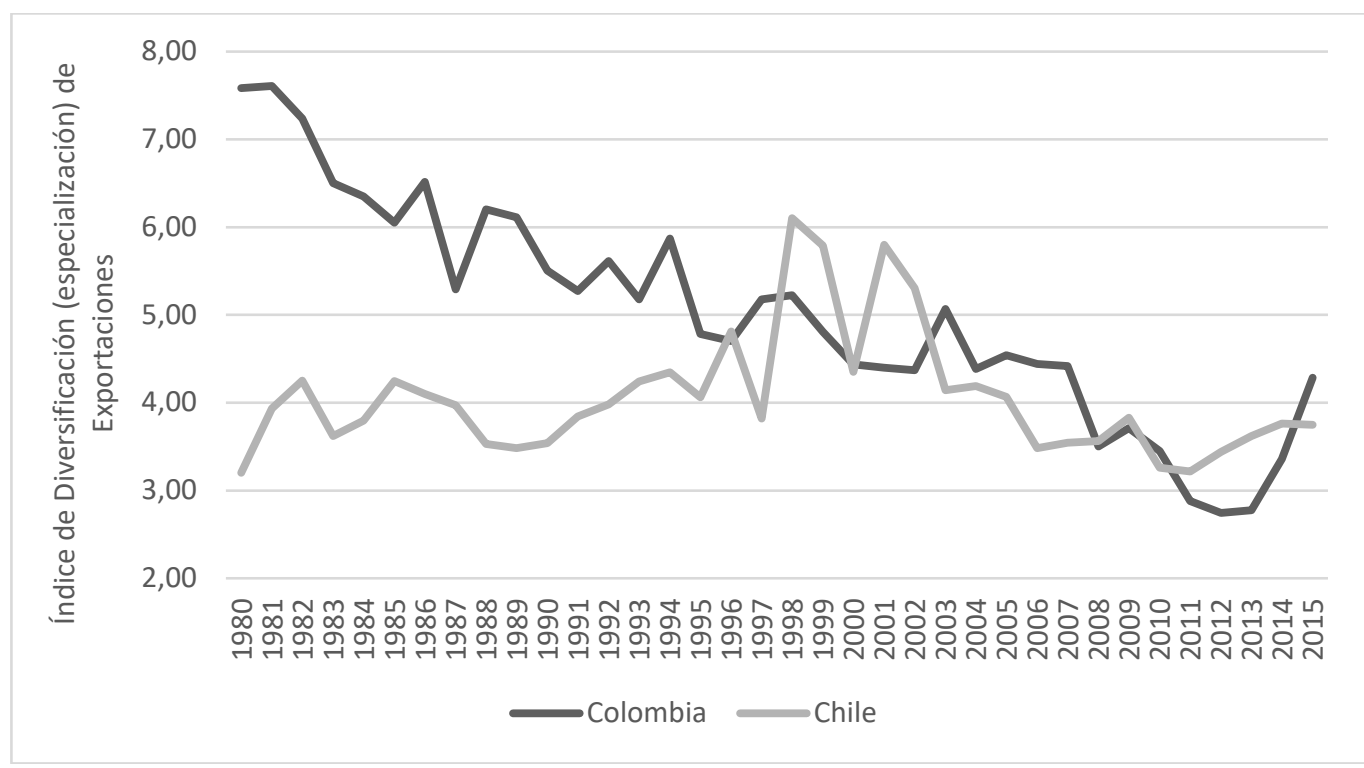

Nota: Valores cercanos a 1 indican especialización, mientras que valores cercanos a 0 indican diversificación.

Fuente: Elaboración propia con base en UNCOMTRADE.

\section{Modelo empírico.}

Para comprobar la hipótesis relacionada con el hecho de que la diversificación de las exportaciones está vinculada con el crecimiento económico a través de externalidades tipo learning by exporting y learning by doing, este documento sigue la modelación sugerida por Herzer y Nowak-Lehnmann (2006). Consideremos entonces una economía con n sectores y Z $\in$ $\mathrm{n}$ sectores exportadores, asumiendo que existe una firma para cada sector y la producción de cada uno $f \in[1, n]$ en cada periodo $t$ está caracterizada por una función neoclásica de producción: 


$$
Y_{f t}=F_{f t}\left(K_{f t}, L_{f t}, W_{t}\right)
$$

donde $Y_{f t}$ es la producción del sector, $K_{f t}$ y $L_{f t}$ representan el capital y el trabajo y $W_{t}$ es un índice del conocimiento público que entra en la función de producción de cada sector $\mathrm{f}$ como una externalidad positiva. La externalidad de conocimiento $W_{t}$ es en su mayoría generado al interior de los sectores exportadores de la economía como resultado de actividades tipo learning by exporting y learning by doing. El proceso learning by exporting se deriva de la ganancia obtenida por los exportadores del conocimiento básico de sus compradores como consumidores foráneos que ofrecen asesoramiento sobre mejoras en la productividad. El proceso learning by doing es asociado con la creación de conocimiento como un derivado de la producción, dependiendo del output acumulado de la firma. Por tanto, una expansión de la firma orientada hacia la exportación incrementa el stock de conocimiento; este proceso de creación también es acelerado por la competencia de mercado. Para simplificar, se asume que cada uno de $\operatorname{los} Z_{t}$ sectores exportadores produce el mismo nivel de conocimiento $W_{e}$, tal que el nivel agregado de conocimiento puede ser descrito por la expresión:

$$
W_{t}=Z_{t} W_{e t}
$$

Dado que $W_{e t}$ es una constante, el nivel de conocimiento de una economía puede expresarse como:

$$
W_{t}=G\left(Z_{t}\right)
$$

Sin embargo, los efectos de aprendizaje pueden depender de la composición de la cesta exportadora: en una base exportadora en la que predominan los productos del sector primario no es muy probable que se difundan efectos learning by doing y learning by exporting, por lo cual se espera que la creación de conocimiento aumente cuando la participación de las manufacturas en las exportaciones ocurre. Por tanto, la externalidad esperada toma la forma:

$$
W_{t}=G\left(Z_{t}, I X_{t}\right)
$$

donde $I X_{t}$ es la proporción de las exportaciones manufactureras y $Z_{t}$ es el número de sectores exportadores, que son tomadas como proxies del stock de conocimiento en la economía.

Además, $W_{t}$ es un bien público que se asume como constante para todos los sectores. Tenemos entonces que los efectos de $W_{t}$ afectan a todos los sectores, pero cómo afecta a $F_{f}$ es desconocido por ellos. Dado $W_{t}, F_{f}$ se comporta como una función con retornos constantes a escala. En condiciones de competencia perfecta y siendo las firmas tomadoras de precios y teniendo:

$$
Y_{t}=\sum_{f=1}^{n} Y_{f t}, \quad K_{t}=\sum_{f=1}^{n} K_{f t}, \quad L_{t}=\sum_{f=1}^{n} L_{f t}
$$

la función de producción total $Y_{t}$ de la economía puede escribirse como:

$$
Y_{t}=\sum_{f=1}^{n} Y_{f t}=F_{t}\left(K_{t}, L_{t}, W_{t}\right)=F_{t}\left(K_{t}, L_{t}\right) G\left(Z_{t}, I X_{t}\right)=K_{t}^{\alpha} L_{t}^{\beta} Z_{t}^{\gamma} I X_{t}^{\delta}
$$

donde $K_{t}$ es el stock de capital acumulado, $L_{t}$ la fuerza laboral de la economía y los parámetros $\alpha, \beta, \gamma$ y $\delta$ son constantes. Al introducir el número de sectores exportadores y la proporción del sector manufacturero en ellas, la ecuación 6 implica que la diversificación horizontal y vertical de las exportaciones están relacionadas con el crecimiento económico a través de las externalidades learning by doing y learning by exporting (si $\gamma, \delta>0)$. Para simplificar, la ecuación 6 puede expresarse como:

$$
\ln Y_{t}=c+\alpha \ln K_{t}+\beta \ln L_{t}+\delta \ln W_{t}+e_{t}
$$


donde $e_{t}$ es el término de error; se asume que sigue un proceso de ruido blanco y es normal e idénticamente distribuido. La forma logarítmica implica que las estimaciones de $\alpha, \beta$ y $\delta$ corresponden a las elasticidades. Por tanto, la hipótesis a probar relacionada con el impacto de la diversificación de las exportaciones sobre el crecimiento es:

$$
\begin{aligned}
& H_{0}=\delta=0 \\
& H_{a}=\delta>0
\end{aligned}
$$

En consecuencia, la hipótesis del crecimiento liderado por la diversificación de las exportaciones no será rechazada si los coeficientes de $\delta$ son positivos y estadísticamente significativos.

\section{Datos y Metodología Econométrica.}

El análisis econométrico se basa en datos anuales que abarcan desde 1980 hasta 2015, obtenidos de las bases de datos del Banco Mundial (BM), de la United Nations Commodity Trade Statistics (UNCOMTRADE), de las Naciones Unidas (UN) y del Fondo Monetario Internacional (FMI).

Siguiendo a Arip, Yee y Bakri (2010), las principales variables de este estudio son el Producto Interno Bruto (PIB) y el Índice de Diversificación de Exportaciones (DSD). E1 Índice de Diversificación de Exportaciones, introducido por Balassa (1989), indica que un valor menor de este índice evidencia un alto nivel de diversificación de exportaciones, mientras que un valor alto del mismo indica un alto nivel de especialización de las exportaciones. El cálculo de este índice se presenta en el Anexo 1.

Como variables de control fueron incluidas: Formación Bruta de Capital (FBC), Inversión Extranjera Directa (IED) y Tasa de Desempleo (TDE). Como se ha mencionado, las economías escogidas para el estudio son las de Chile y Colombia, que atraviesan procesos de profundización de su apertura con marcadas diferencias en su dinámica comercial. Para evaluar la relación de largo plazo entre cada una de las variables se aplican técnicas de cointegración, para lo cual se requiere que las variables sean del mismo orden de integración. En este sentido, primero se realiza para cada variable la prueba de raíz unitaria (orden de integración) y los modelos se especifican con una constante y variable de tendencia; la longitud de los retardos de cada modelo se determina por el Criterio de Información Akaike (AIC).

Posteriormente se utiliza el modelo autorregresivo (VAR), empleado por Johansen y Juselius (1990), para determinar si las variables son cointegradas, que tiene la siguiente forma:

$$
\begin{gathered}
\Delta y_{t}=\sum_{i=1}^{p-1} \Pi_{i} \Delta y_{t-i}-\Pi y_{t-p}+\varepsilon_{t} \\
\varepsilon_{t} \sim I N(0, \Omega), t=1, \ldots, T \\
\Pi=-\left[I-\sum_{i-1}^{p} A_{i}\right] \\
\Pi_{i}=-\left[I-\sum_{j-1}^{i} A_{j}\right]
\end{gathered}
$$


donde $\Delta y$ es un vector $\mathrm{n} \times 1$ de las variables en primera diferencia; $\varepsilon$ es un vector $\mathrm{n} \times 1$ del término del error; $\Pi$ es una matriz $\mathrm{n} \times \mathrm{n}$, en la que $I$ es una matriz identidad $\mathrm{n} \times \mathrm{n}$ y $A$ es una matriz de parámetros $\mathrm{n} \times \mathrm{n}$. El rango de $\Pi$ es el número de raíces características que difieren de cero e indican el número de vectores de cointegración independientes. Para examinar la hipótesis de que las variables son no cointegradas $(\mathrm{r}=0)$ contra la hipótesis alternativa de que las variables tienen uno o más vectores de cointegración ( $\mathrm{r}>0$ ), se calculan los estadísticos Trace y MaxEigen.

$$
\lambda_{\text {trace }}=-T \sum_{i=r+1}^{n} \ln \left(1-\lambda_{i}^{\wedge}\right)
$$

donde $T$ es el número de observaciones utilizables, $\lambda^{\wedge}$ es la raíz característica estimada y In representa el logaritmo natural. Seguidamente se aplica un modelo de vector autorregresivo (VAR) con corrección de errores para determinar las direcciones de causalidad entre las variables. Si las variables son cointegradas, un término de corrección de error se debe especificar en el modelo VAR para hacer las pruebas de causalidad de Granger. Las ecuaciones (8), (9), (10), (11) y (12) son estimadas para examinar causalidad de Granger en el modelo VAR.

$$
\begin{aligned}
& \Delta P I B_{t}=\beta_{0}+\sum_{i=1} \beta_{1} \Delta P I B_{t-i}+\sum_{i=1} \beta_{2} \Delta D S D_{t-i}+\sum_{i=1} \beta_{3} \Delta F B C_{t-i}+\sum_{i=1} \beta_{4} \Delta I E D_{t-i}+ \\
& \sum_{i=1} \beta_{5} \Delta T D E_{t-i}+\beta_{6} E C_{t-1}+\mu_{t}
\end{aligned}
$$

$$
\begin{aligned}
& \Delta D S D_{t}=\beta_{0}+\sum_{i=1} \beta_{1} \Delta D S D_{t-i}+\sum_{i=1} \beta_{2} \Delta P I B_{t-i}+\sum_{i=1} \beta_{3} \Delta F B C_{t-i}+\sum_{i=1} \beta_{4} \Delta I E D_{t-i}+ \\
& \sum_{i=1} \beta_{5} \Delta T D E_{t-i}+\beta_{6} E C_{t-1}+\mu_{t}
\end{aligned}
$$

$$
\begin{aligned}
& \Delta F B C_{t}=\beta_{0}+\sum_{i=1} \beta_{1} \Delta F B C_{t-i}+\sum_{i=1} \beta_{2} \Delta D S D_{t-i}+\sum_{i=1} \beta_{3} \Delta P I B_{t-i}+\sum_{i=1} \beta_{4} \Delta I E D_{t-i}+ \\
& \sum_{i=1} \beta_{5} \Delta T D E_{t-i}+\beta_{6} E C_{t-1}+\mu_{t}
\end{aligned}
$$

$$
\begin{aligned}
& \Delta I E D_{t}=\beta_{0}+\sum_{i=1} \beta_{1} \Delta I E D_{t-i}+\sum_{i=1} \beta_{2} \Delta D S D_{t-i}+\sum_{i=1} \beta_{3} \Delta F B C_{t-i}+\sum_{i=1} \beta_{4} \Delta P I B_{t-i}+ \\
& \sum_{i=1} \beta_{5} \Delta T D E_{t-i}+\beta_{6} E C_{t-1}+\mu_{t}
\end{aligned}
$$

$$
\begin{aligned}
& \Delta T D E_{t}=\beta_{0}+\sum_{i=1} \beta_{1} \Delta T D E_{t-i}+\sum_{i=1} \beta_{2} \Delta D S D_{t-i}+\sum_{i=1} \beta_{3} \Delta F B C_{t-i}+\sum_{i=1} \beta_{4} \Delta I E D_{t-i}+ \\
& \sum_{i=1} \beta_{5} \Delta \mathrm{PIB}+\beta_{6} E C_{t-1}+\mu_{t}
\end{aligned}
$$

donde $E C$ es el término de corrección de errores, $P I B$ el Producto Interno Bruto, $D S D$ el Índice de Diversificación de Exportaciones, $F B C$ la Formación Bruta de Capital, IED la Inversión Extranjera Directa, $T D E$ la Tasa de Desempleo, $\Delta$ indica primera diferencia y $\mu$ los residuos de las ecuaciones.

\section{Resultados empíricos.}

\subsection{Resultados Prueba de Raíz Unitaria.}

A fin de evaluar la relación de largo plazo entre la diversificación de exportaciones y el crecimiento económico para Chile y Colombia usando el enfoque de cointegración, primero se examinan las propiedades de estacionariedad de las series temporales. Esto se hace aplicando la Prueba de Raíz Unitaria desarrollada por Dickey y Fuller (1979).

Antes de aplicar los procedimientos estadísticos se realizó una transformación logarítmica a todas las variables de la serie temporal de cada país del estudio. La prueba ADF de raíz unitaria muestra la existencia de raíces unitarias en las series de Colombia y Chile y, por lo tanto, las 
variables son no estacionarias a nivel, pero después de la primera diferencia se vuelven estacionarias, siendo integradas de orden uno, I (1).

Tabla 1. Resultados del test ADF.

\begin{tabular}{|c|c|c|c|}
\hline \multirow{3}{*}{ País } & \multirow{3}{*}{ Variable } & \multicolumn{2}{|c|}{ ADF Test } \\
\cline { 2 - 4 } & & Nivel & Primera diferencia \\
\hline \multirow{4}{*}{ Chile } & PIB & -1.956254 & $-4.119427^{*}$ \\
\cline { 2 - 4 } & DSD & -3.176850 & $-7.005917^{*}$ \\
\cline { 2 - 4 } & FBC & -2.568098 & $-7.007391 *$ \\
\cline { 2 - 4 } & TDE & -2.211450 & $-5.350110^{*}$ \\
\cline { 2 - 4 } & IED & -2.592526 & $-4.983583 *$ \\
\hline \multirow{4}{*}{ Colombia } & PIB & -2.266483 & $-3.552191 *$ \\
\cline { 2 - 4 } & DSD & $-4.544299 *$ & $-6.197183 *$ \\
\cline { 2 - 4 } & FBC & -2.035938 & $-5.043093^{*}$ \\
\cline { 2 - 4 } & TDE & -1.793016 & $-3.818438^{*}$ \\
\cline { 2 - 4 } & IED & -3.417853 & $-5.652848 *$ \\
\hline
\end{tabular}

Nota: * denota el rechazo de la hipótesis nula al $1 \%$.

Fuente: Elaboración propia con el software Eviews.

\subsection{Resultados Test de Cointegración.}

En este estudio el enfoque de Johansen es utilizado para probar si existe una relación de largo plazo entre la diversificación de las exportaciones y el crecimiento económico para los países seleccionados, usando las variables PIB, DSD, FBC, IED, TDE, que son integradas de orden I(1). Para la realización del test de Johansen se hizo necesario determinar el número de retardos, de modo que se tomó el criterio de información de Akaike. De acuerdo con las estimaciones, en el caso chileno se emplearon dos retardos, mientras que para Colombia solo se usó uno (Tabla 2).

Tabla 2. Identificación de los retardos.

\begin{tabular}{|c|c|c|c|c|c|c|}
\hline & Retardos & LR & FPE & AIC & SC & HQ \\
\hline \multirow{4}{*}{ Chile } & 0 & NA & $4.98 \mathrm{e}-10$ & -7.232028 & $-7.005285^{*}$ & $-7.155736^{*}$ \\
\cline { 2 - 7 } & 1 & 47.38401 & $4.00 \mathrm{e}-10$ & -7.471840 & -6.111379 & -7.014086 \\
\cline { 2 - 7 } & 2 & $38.62900^{*}$ & $3.51 \mathrm{e}-10^{*}$ & $-7.712552^{*}$ & -5.218373 & -6.873337 \\
\hline \multirow{3}{*}{ Colombia } & 0 & NA & $2.97 \mathrm{e}-07$ & -0.838939 & -0.614474 & -0.762390 \\
\cline { 2 - 7 } & 1 & $278.8642^{*}$ & $6.23 \mathrm{e}-11^{*}$ & $-9.327785^{*}$ & $-7.980996^{*}$ & $-8.868491^{*}$ \\
\cline { 2 - 7 } & 2 & 27.96879 & $8.90 \mathrm{e}-11$ & -9.073231 & -6.604118 & -8.231193 \\
\hline
\end{tabular}

Nota: *denota el retardo óptimo definido por cada criterio.

LR: sequential modified LR test statistic (each test at 5\% level)

FPE: Final prediction error

AIC: Akaike information criterion

SC: Schwarz information criterion

HQ: Hannan-Quinn information criterion

Fuente: Elaboración propia con el software Eviews.

Los estadísticos Trace y Max-Eigen sugieren que existe cointegración entre las variables para Chile y Colombia. Por lo anterior, las variables se mueven juntas en el largo plazo y sus desviaciones del equilibrio serán corregidas en el largo plazo. La Tabla 3 muestra los resultados de la prueba de Cointegración de Johansen.

Los parámetros estimados de largo plazo, obtenidos del Test de Johansen, muestran resultados distintos para Colombia y Chile. En este sentido, para Colombia el DSD mostró una 
relación positiva con el PIB, mientras que para Chile la relación fue negativa, en línea con la relación encontrada por Imbs y Wacziarg (2003) (ver Anexo 2).

Tabla 3. Resultados del Test de Cointegración de Johansen.

\begin{tabular}{|c|c|c|c|c|c|c|c|}
\hline País & $\begin{array}{l}\text { Hipótesis } \\
\text { Nula }\end{array}$ & $\begin{array}{c}\text { Trace } \\
\text { Statistic }\end{array}$ & $5 \% \mathrm{CV}$ & Prob. & $\begin{array}{c}\text { Max- } \\
\text { Eigen } \\
\text { Statistic }\end{array}$ & $5 \% \mathrm{CV}$ & Prob. \\
\hline \multirow{6}{*}{ COLOMBIA } & Ho: $r=0$ & 121.1292 & 95.75366 & $0.0003 *$ & 47.41757 & 40.07757 & $0.0063 *$ \\
\hline & Ho: $r \leq 1$ & 73.71161 & 69.81889 & $0.0236^{*}$ & 32.69030 & 33.87687 & 0.0688 \\
\hline & Ho: $r \leq 2$ & 41.02130 & 47.85613 & 0.1880 & 22.69644 & 27.58434 & 0.1868 \\
\hline & Ho: $r \leq 3$ & 18.32486 & 29.79707 & 0.5422 & 10.86595 & 21.13162 & 0.6607 \\
\hline & Ho: $r \leq 4$ & 7.458909 & 15.49471 & 0.5249 & 7.457455 & 14.26460 & 0.4365 \\
\hline & Ho: $r \leq 5$ & 0.001453 & 3.841466 & 0.9680 & 0.001453 & 3.841466 & 0.9680 \\
\hline \multirow{5}{*}{ CHILE } & Ho: $r=0$ & 120.6901 & 69.81889 & $0.0000 *$ & 50.56936 & 33.87687 & $0.0002 *$ \\
\hline & Ho: $r \leq 1$ & 70.12070 & 47.85613 & $0.0001 *$ & 31.23979 & 27.58434 & $0.0162 *$ \\
\hline & Ho: $r \leq 2$ & 38.88091 & 29.79707 & $0.0034 *$ & 17.66235 & 21.13162 & 0.1430 \\
\hline & Ho: $r \leq 3$ & 21.21856 & 15.49471 & $0.0061 *$ & 12.31143 & 14.26460 & 0.0995 \\
\hline & Ho: $r \leq 4$ & 8.907131 & 3.841466 & $0.0028 *$ & 8.907131 & 3.841466 & $0.0028 *$ \\
\hline
\end{tabular}

\subsection{Test de Causalidad de Granger.}

A continuación, se examinan las relaciones causales y sus direcciones entre las variables del estudio para lo que se aplicó el test de causalidad de Granger. Dado que tanto para Chile como para Colombia se probó la existencia de vectores de cointegración entre las variables, la dinámica de interacciones causales entre las variables debe ser expresada en forma de vector de corrección de errores (VEC). Esto permite medir separadamente las relaciones causales en el corto plazo y largo plazo.

La Tabla 4 presenta el resumen del Test de Causalidad de Granger para ambos países. Como se puede observar, en Chile se comprueba una relación causal de doble vía entre crecimiento económico y concentración de exportaciones; en este caso, los resultados son coherentes con la tesis sostenida por Imbs y Wacziarg (2003) y Cadot, Carrere y Strauss (2011), en tanto que, en las economías con altos niveles de ingreso, se estimula la re-concentración de las exportaciones, privilegiando los productos en donde tienen una mayor ventaja comparativa (Gozgor \& Can, 2016). De igual modo, se evidenció causalidad a la Granger que va desde el PIB a la inversión extranjera directa (IED), así como también relaciones en doble vía entre formación bruta de capital (FBC) y la concentración de las exportaciones (DSD), entre DSD y tasa de desempleo (TDE) y, por último, causalidades unidireccionales que van desde FBC a IED, y de TDE a IED.

Para Colombia, los resultados soportan una causalidad en sentido de Granger desde el crecimiento hacia la diversificación de las exportaciones, así como también para IED. 
Tabla 4. Resultados del test de Causalidad de Granger.

\begin{tabular}{|c|c|c|c|c|c|}
\hline \multicolumn{3}{|l|}{ Chile } & \multicolumn{3}{|l|}{ Colombia } \\
\hline Null Hypothesis: & F-Statistic & Prob. & Null Hypothesis: & F-Statistic & Prob. \\
\hline DLDSD does not Granger Cause DLNPIB & 4.52711 & $0.0414^{*}$ & LNDSD does not Granger Cause LNPIB & 0.3862 & 0.5387 \\
\hline DLNPIB does not Granger Cause DLDSD & 4.21132 & $0.0487^{*}$ & LNPIB does not Granger Cause LNDSD & 5.44571 & $0.0261 *$ \\
\hline DLNIED does not Granger Cause DLNPIB & 0.00998 & 0.9211 & LNIED does not Granger Cause LNPIB & 0.04106 & 0.8407 \\
\hline DLNPIB does not Granger Cause DLNIED & 6.55912 & $0.0155^{*}$ & LNPIB does not Granger Cause LNIED & 8.67682 & $0.006^{*}$ \\
\hline DLNFBC does not Granger Cause DLNPIB & 1.58208 & 0.2179 & LNFBC does not Granger Cause LNPIB & 0.00142 & 0.9702 \\
\hline DLNPIB does not Granger Cause DLNFBC & 0.05988 & 0.8083 & LNPIB does not Granger Cause LNFBC & 1.82889 & 0.1857 \\
\hline DLNTDE does not Granger Cause DLNPIB & 0.93129 & 0.342 & LNTDE does not Granger Cause LNPIB & 0.00673 & 0.9351 \\
\hline DLNPIB does not Granger Cause DLNTDE & $2.80 \mathrm{E}-05$ & 0.9958 & LNPIB does not Granger Cause LNTDE & 0.0257 & 0.8736 \\
\hline DLNIED does not Granger Cause DLDSD & 1.49579 & 0.2305 & LNIED does not Granger Cause LNDSD & 2.86211 & 0.1004 \\
\hline DLDSD does not Granger Cause DLNIED & 0.00028 & 0.9867 & LNDSD does not Granger Cause LNIED & 4.13279 & 0.0504 \\
\hline DLNFBC does not Granger Cause DLDSD & 4.20801 & $0.0488^{*}$ & LNFBC does not Granger Cause LNDSD & 0.00866 & 0.9264 \\
\hline DLDSD does not Granger Cause DLNFBC & 4.8141 & $0.0358^{*}$ & LNDSD does not Granger Cause LNFBC & 2.12917 & 0.1543 \\
\hline DLNTDE does not Granger Cause DLDSD & 4.66083 & $0.0387^{*}$ & LNTDE does not Granger Cause LNDSD & 1.1488 & 0.2918 \\
\hline DLDSD does not Granger Cause DLNTDE & 6.66155 & $0.0148^{*}$ & LNDSD does not Granger Cause LNTDE & 0.86691 & 0.3588 \\
\hline DLNFBC does not Granger Cause DLNIED & 11.56 & $0.0019^{*}$ & LNFBC does not Granger Cause LNIED & 0.52865 & 0.4725 \\
\hline DLNIED does not Granger Cause DLNFBC & 0.3541 & 0.5561 & LNIED does not Granger Cause LNFBC & 1.10918 & 0.3001 \\
\hline DLNTDE does not Granger Cause DLNIED & 11.5598 & $0.0019^{*}$ & LNTDE does not Granger Cause LNIED & 0.25338 & 0.6181 \\
\hline DLNIED does not Granger Cause DLNTDE & 0.12784 & 0.7231 & LNIED does not Granger Cause LNTDE & 0.03947 & 0.8438 \\
\hline DLNTDE does not Granger Cause DLNFBC & 0.48166 & 0.4928 & LNTDE does not Granger Cause LNFBC & 0.0385 & 0.8457 \\
\hline DLNFBC does not Granger Cause DLNTDE & 0.69142 & 0.4120 & LNFBC does not Granger Cause LNTDE & 0.49929 & 0.4849 \\
\hline
\end{tabular}




\section{Conclusiones.}

En este artículo se examinó empíricamente la relación de corto y largo plazo entre la diversificación de exportaciones y el crecimiento económico de Colombia y Chile. Se usaron datos anuales desde 1980 hasta 2015 y se emplearon técnicas de series de tiempo para estudiar las relaciones entre las variables y sus interacciones dinámicas, usando el Test de Cointegración de Johansen y el Test de Causalidad de Granger.

Los resultados indican la existencia de vectores de cointegración entre las variables para Colombia y para Chile, lo que implica que estas variables se desplazan juntas en el largo plazo y sus desviaciones de la trayectoria de equilibrio de largo plazo serán corregidas. Sin embargo, se encontró evidencia de la no existencia de causalidad unidireccional que va desde el Índice de Diversificación de Exportaciones hacia el Producto Interno Bruto para ambas economías, con la marcada diferencia de que en el caso de Colombia la relación va del crecimiento a la diversificación de exportaciones.

En el caso de Colombia, los resultados permiten inferir que a pesar de que las reformas de apertura ya han sido aplicadas, la política de promoción de exportaciones ha desarrollado poco los sectores no tradicionales, de tal forma que sus contribuciones al crecimiento económico no han sido significativas. Las políticas de diversificación no han tenido los alcances esperados y no se ha determinado un marco de largo plazo en el que se establezcan las acciones e incentivos que el Estado debe proveer para el desarrollo de nuevos sectores exportadores. No obstante, la implementación de políticas sectoriales, los objetivos y los marcos de política cambian en el tiempo, de acuerdo con la ruta trazada por cada periodo presidencial, teniendo entonces un problema de inconsistencia dinámica. Por tanto, si se quiere alcanzar un mayor grado de convergencia con economías más avanzadas a través de la diversificación de la cesta exportadora, se requiere adoptar estrategias que incentiven el descubrimiento de nuevas actividades.

Es importante destacar que, a partir de este trabajo, para el caso colombiano es posible desarrollar una posterior indagación relacionada con el efecto de las exportaciones no tradicionales en el crecimiento económico teniendo en cuenta que gran parte de la composición de la cesta exportadora de este país está dominada por las exportaciones de petróleo y derivados, y carbón.

\section{Referencias}

Abdulai, A., \& Jaquet, P. (2002). Exports and Growth: Cointegration and Causality Evidence for Côte d'Ivoire. African Development Bank, Blackwell Publishers, 14(1), 1-17.

Acemoglu, D., \& Zilibotti, F. (1997). Was Prometheus unbound by chance? Risk, diversification, and growth. Journal of Political Economy, 105(4), 709-751.

Agosin, M. (1999). Trade and growth in Chile. CEPAL Review, 68, 79-100.

Al-Marhubi, F. (2000). Export diversification and growth: an empirical investigation. Applied Economics Letters, 7(9), 559-562.

Arip, M., Yee, L., \& Bakri, K. (2010). Export Diversification and Economic Growth in Malaysia. MPRA Paper, 20588, Unimas, Reitaku University.

Arrow, K. (1962). The economic implications of learning by doing. Review of Economic Studies, 29(3), 153-173.

Awokuse, T. (2003). Is the export-led growth hypothesis valid for Canada? Canadian Journal of Economics/Revue canadienne d'économique, 36(1), 126-136. 
Balaguer, J., \& Cantavella-Jordá, M. (2004). Structural change in exports and economic growth: cointegration and causality analysis for Spain (1961-2000). Applied Economics, 36(5), 473-477.

Balassa, B. (Ed.). (1989). Trade Liberalization and 'Revealed' Comparative Advantage. Hertfordshire: Harvester Wheatsheaf.

Bleaney, M., \& Greenaway D. (2001). The impact of terms of trade and real exchange rate volatility on investment and growth in sub-Saharan Africa. Journal of Development Economics, 65(2), 491500 .

Cadot, O., Carrere, C., \& Strauss-Kahn, V. (2011). Export diversification: what's behind the hump? Review of Economic and Statistics, 93(2), 590-605.

Chen, S. (2007). Exactly what is the link between export and growth in Taiwan? New evidence from the Granger causality test. Economics Bulletin, 6(7), 1-10.

Dawe, D. (1996). A new look at the effects of export instability on investment and growth. World Development, 24(12), 1905-1914.

Dickey, D., \& Fuller, W. (1979). Distribution of the estimators for autoregressive time series with a unit root. Journal of the American Statistical Association, 74(366), 427-431.

García, P., Meller P., \& Repetto, A. (1996). Las exportaciones como motor del crecimiento: la evidencia chilena. En Patricio Meller (comp.), El modelo exportador chileno: crecimiento y equidad, Santiago de Chile, Corporación de Investigaciones Económicas para Latinoamérica (CIEPLAN), pp. 19-42.

Gokmenoglu, K., Sehnaz, Z., \& Taspinar, N. (2015). The Export-Led Growth: A Case Study of Costa Rica. Procedia Economics and Finance, 25, 471-477.

Gozgor, G., \& Can, M. (2016). Effects of the product diversification of exports on income at different stages of economic development. Eurasian Buisiness Review, 6(2), 215-235.

Gutiérrez de Piñeres, S., \& Ferrantino, M. (1997). Export diversification and structural dynamics in the growth process: The case of Chile. Journal of development Economics, 52(2), 375-391.

Gutiérrez de Piñeres, S., \& Ferrantino, M. (1999). Export sector dynamics and domestic growth: The case of Colombia. Review of Development Economics, 3(3), 268-280.

Hausmann, R., \& Rodrik, D. (2003). Economic development as self-discovery. Journal of Development Economics, 72(2), 603-633.

Hausmann, R., Hwang, J., \& Rodrik, D. (2007). What you export matters. Journal of Economic Growth, $12(1), 1-25$.

Hausmann, R., \& Klinger, B. (2006). The evolution of comparative advantage: the impact of the structure of the product space. Center for International Development and Kennedy School of Government Harvard University.

Herzer, D., \& Nowak-Lehnmann, F. (2006). ¿What does export diversification do for growth? An econometric analysis. Applied Economics, 38(15), 1825-1838. 
Hye, Q., Wizarat, S., \& Lau, W. (2013). Trade-led growth hypothesis: An empirical analysis of South Asian countries, Economic Modelling, 35, 654-660.

Imbs, J., \& Wacziarg, R. (2003). Stages of Diversification. American Economic Review, 93(1), 63-86.

Jin, J. (2002). Exports and Growth: is the Export-led Growth Hypothesis valid for provincial economies? Applied Economics Letters, 34(1), 63-76.

Johansen, S., \& Juselius, K. (1990). Maximum likelihood estimation and inference on cointegration with applications to the demand for money. Oxford Bulletin of Economics and Statistics, 52(2), 169-210.

Mejía, J. (2011). Export Diversification, International Trade, and Economic Growth: A Survey of the Literature. In Export Diversification and Economic Growth, Physica-Verlag HD, pp. 9-47.

Michaely, M. (1977). Exports and growth: an empirical investigation. Journal of Development Economics, 4(1), 49-53.

Misztal, P. (2011). Export diversification and economic growth in European Union member states, Acta Oeconomia, 10(2), 55-64.

Mordecki, G., \& Piaggio, M. (2008). Integración regional: ¿el crecimiento económico a través de la diversificación de exportaciones? Documentos de Trabajo, Instituto de Economía - IECON.

Moschos, D. (1989). Export expansion, growth and the level of economic development: an empirical analysis. Journal of Development Economics, 30(1), 93-102.

Panas, E., \& Vamvoukas, G. (2002). Further evidence on the export-led growth hypothesis. Applied Economics Letters, 9(11), 731-735.

Romer, P. (1990). Endogenous Technological Change. The Journal of Political Economy, 98(5), S71S102.

Ruiz, M., \& Vera, R. (2013). Exportaciones no tradicionales 2002-2012: Una historia de crecimiento, apertura y diversificación. Revista Moneda, 156, 33-36.

Sannassee, R., Seetanah, B., \& Lamport, M. (2014). Export diversification and economic growth: the case of Mauritius. In Connecting to global markets, Challenges and opportunities: case studies presented by WTO chair-holders. WTO Publications.

Stanley, D., \& Bunnag, S. (2001). A new look at the benefits of diversification: lessons from Central America. Applied Economics, 33(11), 1369-1383. 


\section{ANEXO 1. Formulación del índice de diversificación de exportaciones DSD.}

Este índice, introducido por Balassa (1989), se calcula tomando como base la siguiente ecuación:

$$
\text { a) } \quad \sigma=\sqrt{\frac{1}{N} \sum_{i=1}^{N}(V C R-\overline{V C} \bar{R})^{2}}
$$

donde $N$ es el número de productos, $V C R$ es el índice de ventaja comparativa revelada de un producto y $\overline{V C R}$ es la media del $V C R$ de $N$ número de productos.

Particularmente, este método utiliza la desviación estándar del Índice de Ventaja Comparativa Revelada $(V C R)$. La ecuación (a) indica que un valor menor del índice evidencia un alto nivel de diversificación de exportaciones, mientras que un valor alto en el índice indica un alto nivel de especialización de las exportaciones. La ecuación (b), muestra la notación matemática del índice $V C R$.

$$
\text { b) } \quad V C R_{i}^{j}=\frac{\left(x_{i}^{j} / x_{t}^{j}\right)}{\left(X_{i}^{w} / X_{t}^{w}\right)}
$$

donde $V C R$ es el Índice de Ventaja Comparativa Revelada, $X_{i}^{j}$ son las exportaciones del producto $i$ del país $j, X_{t}^{j}$ son las exportaciones totales del país $j, X_{i}^{w}$ son las exportaciones del producto $i$ en el mundo y $X_{t}^{w}$ son las exportaciones totales del mundo.

ANEXO 2. Vectores de Cointegración encontrados para Chile.

\begin{tabular}{ccccc} 
1 Cointegrating Equation(s): & Log likelihood & 138.4531 & \\
\hline \hline & & & \\
Normalized cointegrating coefficients (standard error in parentheses) & \\
DLNDSD & DLNFBC & DLNIED & DLNPIB & DLNTED \\
1.000000 & -6.829638 & 0.934533 & -6.180710 & -3.443501 \\
& $(0.72357)$ & $(0.17075)$ & $(2.17123)$ & $(0.67553)$
\end{tabular}

Adjustment coefficients (standard error in parentheses)

\begin{tabular}{|c|c|c|c|c|}
\hline \multicolumn{2}{|c|}{2 Cointegrating Equation(s): } & Log likelihood & \multicolumn{2}{|l|}{155.0088} \\
\hline \multicolumn{5}{|c|}{ Normalized cointegrating coefficients (standard error in parentheses) } \\
\hline DLNDSD & DLNFBC & DLNIED & DLNPIB & DLNTED \\
\hline 1.000000 & 0.000000 & $\begin{array}{l}2.725762 \\
(1.40676)\end{array}$ & $\begin{array}{l}29.53555 \\
(17.5540)\end{array}$ & $\begin{array}{r}-11.53346 \\
(4.50720)\end{array}$ \\
\hline 0.000000 & 1.000000 & $\begin{array}{l}0.262273 \\
(0.21238)\end{array}$ & $\begin{array}{l}5.229597 \\
(2.65010)\end{array}$ & $\begin{array}{r}-1.184536 \\
(0.68044)\end{array}$ \\
\hline
\end{tabular}

\begin{tabular}{lr} 
D(DLNDSD) & -0.057499 \\
& $(0.05462)$ \\
D(DLNFBC) & 0.203440 \\
& $(0.03770)$ \\
D(DLNIED) & -0.418272 \\
& $(0.15419)$ \\
D(DLNPIB) & 0.019746 \\
& $(0.00996)$ \\
D(DLNTED) & -0.060043 \\
& $(0.05646)$ \\
\hline
\end{tabular}

Adjustment coefficients (standard error in parentheses)

$\begin{array}{lll}\mathrm{D}(\mathrm{DLNDSD}) & -0.021680 & 0.240102 \\ & (0.06168) & (0.38720)\end{array}$




\begin{tabular}{|c|c|c|c|c|}
\hline D(DLNFBC) & $\begin{array}{c}0.128273 \\
(0.03241)\end{array}$ & $\begin{array}{r}-1.069197 \\
(0.20344)\end{array}$ & & \\
\hline $\mathrm{D}(\mathrm{DLNIED})$ & $\begin{array}{r}-0.558868 \\
(0.16994)\end{array}$ & $\begin{array}{c}3.455614 \\
(1.06675)\end{array}$ & & \\
\hline D(DLNPIB) & $\begin{array}{c}0.000958 \\
(0.00892)\end{array}$ & $\begin{array}{r}-0.054815 \\
(0.05602)\end{array}$ & & \\
\hline D(DLNTED) & $\begin{array}{l}0.057448 \\
(0.04673)\end{array}$ & $\begin{array}{r}-0.090464 \\
(0.29331)\end{array}$ & & \\
\hline \multicolumn{2}{|c|}{3 Cointegrating Equation(s): } & Log likelihood & 168.8290 & \\
\hline \multicolumn{5}{|c|}{ Normalized cointegrating coefficients (standard error in parentheses) } \\
\hline \multirow{2}{*}{$\begin{array}{r}\text { DLNDSD } \\
1.000000\end{array}$} & DLNFBC & DLNIED & DLNPIB & DLNTED \\
\hline & 0.000000 & 0.000000 & $\begin{array}{c}0.278048 \\
(0.47193)\end{array}$ & $\begin{array}{r}-0.101263 \\
(0.12893)\end{array}$ \\
\hline 0.000000 & 1.000000 & 0.000000 & $\begin{array}{l}2.414441 \\
(0.93512)\end{array}$ & $\begin{array}{r}-0.084531 \\
(0.25547)\end{array}$ \\
\hline 0.000000 & 0.000000 & 1.000000 & $\begin{array}{l}10.73370 \\
(6.03996)\end{array}$ & $\begin{array}{r}-4.194128 \\
(1.65008)\end{array}$ \\
\hline
\end{tabular}

Adjustment coefficients (standard error in parentheses)

$\begin{array}{lccc}\text { D(DLNDSD) } & -1.641080 & 0.275356 & 0.006655 \\ & (0.33741) & (0.28087) & (0.05117) \\ \text { D(DLNFBC) } & 0.181579 & -1.070357 & 0.069130 \\ & (0.24425) & (0.20332) & (0.03704) \\ \text { D(DLNIED) } & 0.823824 & 3.425514 & -0.619400 \\ & (1.25241) & (1.04253) & (0.18994) \\ \text { D(DLNPIB) } & -0.038497 & -0.053956 & -0.011699 \\ & (0.06687) & (0.05566) & (0.01014) \\ \text { D(DLNTED) } & 0.137263 & -0.092201 & 0.132726 \\ & (0.35212) & (0.29311) & (0.05340)\end{array}$

\begin{tabular}{ccccc}
\hline \hline 4 Cointegrating Equation(s): & Log likelihood & 178.6006 & \\
\hline \hline \multirow{2}{*}{\begin{tabular}{c}
\multirow{2}{*}{ Normalized cointegrating coefficients (standard error in parentheses) } \\
DLNDSD
\end{tabular}} & DLNFBC & DLNIED & DLNPIB & DLNTED \\
1.000000 & 0.000000 & 0.000000 & 0.000000 & 0.031067 \\
& & & & $(0.09291)$ \\
0.000000 & 1.000000 & 0.000000 & 0.000000 & 1.064563 \\
& & & & $(0.11549)$ \\
0.000000 & 0.000000 & 1.000000 & 0.000000 & 0.914313 \\
& & & & $(0.47002)$ \\
0.000000 & 0.000000 & 0.000000 & 1.000000 & -0.475926 \\
& & & & $(0.10093)$ \\
& & & & \\
Adjustment coefficients (standard error in parentheses) & & \\
D(DLNDSD) & -1.676686 & 0.071980 & -0.077807 & 0.310951 \\
& $(0.32349)$ & $(0.29863)$ & $(0.07302)$ & $(0.28530)$ \\
D(DLNFBC) & 0.167749 & -1.149356 & 0.036322 & -1.779769 \\
& $(0.24183)$ & $(0.22325)$ & $(0.05459)$ & $(0.21329)$ \\
D(DLNIED) & 0.566708 & 1.956866 & -1.229327 & 2.075050 \\
& $(1.03282)$ & $(0.95345)$ & $(0.23315)$ & $(0.91090)$ \\
D(DLNPIB) & -0.035217 & -0.035220 & -0.003917 & -0.269405 \\
& $(0.06641)$ & $(0.06131)$ & $(0.01499)$ & $(0.05857)$ \\
D(DLNTED) & 0.085552 & -0.387574 & 0.010058 & 1.285191 \\
& $(0.32248)$ & $(0.29770)$ & $(0.07280)$ & $(0.28442)$ \\
\hline \hline
\end{tabular}

Fuente: Elaboración propia con el software Eviews. 


\section{Vectores de cointegración encontrados para Colombia.}

1 Cointegrating Equation(s): $\quad$ Log likelihood $\quad 192.0847$

$\begin{array}{ccccc}\text { Normalized cointegrating coefficients } & \text { (standard error in parentheses) } & \\ \text { LNPIB } & \text { LNDSD } & \text { LNFBC } & \text { LNIED } & \text { LNTDE } \\ 1.000000 & -0.105686 & 0.675506 & -0.418502 & 0.524262 \\ & (0.27314) & (0.32757) & (0.06627) & (0.15385)\end{array}$

Adjustment coefficients (standard error in parentheses)

$\begin{array}{lr}\text { D(LNPIB) } & 0.025649 \\ & (0.02163) \\ \text { D(LNDSD) } & -0.047420 \\ & (0.14295) \\ \text { D(LNFBC) } & 0.054490 \\ & (0.13069) \\ \text { D(LNIED) } & 2.218368 \\ & (0.50093) \\ \text { D(LNTDE) } & -0.183440 \\ & (0.12819)\end{array}$

\begin{tabular}{ccccc}
\hline \hline 2 Cointegrating Equation(s): & Log likelihood & \multicolumn{2}{c}{200.1398} & \\
\hline \hline & Normalized cointegrating coefficients & (standard error in parentheses) & \\
LNPIB & LNDSD & LNFBC & LNIED & LNTDE \\
1.000000 & 0.000000 & 0.709642 & -0.395361 & 0.495858 \\
& & $(0.30948)$ & $(0.03930)$ & $(0.14437)$ \\
0.000000 & \multirow{2}{*}{1.000000} & 0.323001 & 0.218968 & -0.268758 \\
& & $(0.33751)$ & $(0.04286)$ & $(0.15745)$
\end{tabular}

Adjustment coefficients (standard error in parentheses)

$\begin{array}{lcr}\text { D(LNPIB) } & 0.003202 & -0.040137 \\ & (0.02783) & (0.03044) \\ \text { D(LNDSD) } & -0.236619 & -0.310449 \\ & (0.18066) & (0.19762) \\ \text { D(LNFBC) } & -0.184233 & -0.403793 \\ & (0.15792) & (0.17275) \\ \text { D(LNIED) } & 2.584746 & 0.376430 \\ & (0.65359) & (0.71497) \\ \text { D(LNTDE) } & 0.126002 & 0.535334 \\ & (0.14301) & (0.15644)\end{array}$

\begin{tabular}{ccccc}
\hline \hline 3 Cointegrating Equation(s): & Log likelihood & \multicolumn{2}{c}{205.6287} & \\
\hline \hline \multicolumn{2}{l}{ Normalized cointegrating coefficients } & (standard error & in parentheses) & \\
LNPIB & LNDSD & LNFBC & LNIED & LNTDE \\
1.000000 & 0.000000 & 0.000000 & -0.347654 & 0.393251 \\
& & & $(0.03037)$ & $(0.12312)$ \\
0.000000 & 1.000000 & 0.000000 & 0.240682 & -0.315461 \\
& & & $(0.02700)$ & $(0.10947)$ \\
0.000000 & 0.000000 & 1.000000 & -0.067226 & 0.144589 \\
& & & $(0.02673)$ & $(0.10836)$
\end{tabular}

Adjustment coefficients (standard error in parentheses)

$\begin{array}{lccc}\text { D(LNPIB) } & -0.005302 & -0.050904 & -0.002933 \\ & (0.03700) & (0.04337) & (0.03483) \\ \text { D(LNDSD) } & -0.558223 & -0.717615 & 0.025227 \\ & (0.22181) & (0.26002) & (0.20881) \\ \text { D(LNFBC) } & -0.045566 & -0.228234 & -0.387678 \\ & (0.20655) & (0.24213) & (0.19444)\end{array}$




$\begin{array}{cccc}\mathrm{D}(\text { LNIED }) & 2.731845 & 0.562664 & 1.821626 \\ & (0.87000) & (1.01985) & (0.81901) \\ \mathrm{D}(\mathrm{LNTDE}) & 0.187496 & 0.613188 & 0.206226 \\ & (0.18975) & (0.22243) & (0.17863)\end{array}$

\begin{tabular}{|c|c|c|c|c|}
\hline \multicolumn{2}{|c|}{4 Cointegrating Equation(s): } & Log likelihood & \multicolumn{2}{|l|}{209.2215} \\
\hline \multicolumn{5}{|c|}{ Normalized cointegrating coefficients (standard error in parentheses) } \\
\hline LNPIB & LNDSD & LNFBC & LNIED & LNTDE \\
\hline 1.000000 & 0.000000 & 0.000000 & 0.000000 & $\begin{array}{r}-1.117360 \\
(0.36540)\end{array}$ \\
\hline 0.000000 & 1.000000 & 0.000000 & 0.000000 & $\begin{array}{l}0.730341 \\
(0.26428)\end{array}$ \\
\hline 0.000000 & 0.000000 & 1.000000 & 0.000000 & $\begin{array}{r}-0.147517 \\
(0.12889)\end{array}$ \\
\hline 0.000000 & 0.000000 & 0.000000 & 1.000000 & $\begin{array}{r}-4.345153 \\
(1.14048)\end{array}$ \\
\hline \multicolumn{5}{|c|}{ Adjustment coefficients (standard error in parentheses) } \\
\hline D(LNPIB) & $\begin{array}{r}-0.013962 \\
(0.04043)\end{array}$ & $\begin{array}{r}-0.050505 \\
(0.04317)\end{array}$ & $\begin{array}{l}0.002380 \\
(0.03614)\end{array}$ & $\begin{array}{r}-0.009454 \\
(0.00889)\end{array}$ \\
\hline D(LNDSD) & $\begin{array}{r}-0.430269 \\
(0.23614)\end{array}$ & $\begin{array}{r}-0.723504 \\
(0.25215)\end{array}$ & $\begin{array}{r}-0.053265 \\
(0.21111)\end{array}$ & $\begin{array}{l}0.008460 \\
(0.05194)\end{array}$ \\
\hline $\mathrm{D}(\mathrm{LNFBC})$ & $\begin{array}{l}0.018236 \\
(0.22484)\end{array}$ & $\begin{array}{r}-0.231170 \\
(0.24007)\end{array}$ & $\begin{array}{r}-0.426817 \\
(0.20101)\end{array}$ & $\begin{array}{r}-0.018611 \\
(0.04946)\end{array}$ \\
\hline D(LNIED) & $\begin{array}{l}3.121290 \\
(0.93788)\end{array}$ & $\begin{array}{l}0.544741 \\
(1.00144)\end{array}$ & $\begin{array}{l}1.582724 \\
(0.83846)\end{array}$ & $\begin{array}{r}-0.970849 \\
(0.20630)\end{array}$ \\
\hline D(LNTDE) & $\begin{array}{l}0.266012 \\
(0.20511)\end{array}$ & $\begin{array}{l}0.609575 \\
(0.21901)\end{array}$ & $\begin{array}{l}0.158061 \\
(0.18337)\end{array}$ & $\begin{array}{l}0.061666 \\
(0.04512)\end{array}$ \\
\hline
\end{tabular}

Fuente: Elaboración propia con el software Eviews. 